4. Meyer-RochowGY, Gifford A, Samra J, et al. The intrapancreatic splenunculus. Am J Surg 2007; 194; 75
5. Brasca L, Zanello A, De Gaspari A, et al. Intrapancreatic accessory spleen mimicking a neuroendocrine tumour: magnetic resonance findings and possible diagnostic role of different nuclear medicine tests. Eur Radiol 2004; 14: 1322.

\title{
Elevated Everolimus Levels During Severe Diarrhea in Two Children After Heart Transplantation
}

$\mathrm{M}$ inimization of side effects of immunosuppressive therapy after pediatric heart transplantation is an important objective of aftercare. Not only various medications and foods but also gastrointestinal problems can cause increased peak levels of immunosuppressants. Especially, tacrolimus is well known for elevated levels during diarrhea (1-3). In the follow-up care of pediatric heart transplanted patients, the new immunosuppressant everolimus is increasingly used. However, the influence of diarrhea on everolimus blood levels has not been evaluated yet. We report about two patients with elevated everolimus levels during severe diarrhea with at least two watery stools per day.

\section{CASE 1}

The 3.5-year-old girl with history of heart transplantation in June 2010 because of noncompaction cardiomyopathy was on immunosuppressive therapy with everolimus and tacrolimus. The target value of tacrolimus was $6 \mathrm{ng} / \mathrm{mL}$; the one of everolimus ranged from 4 to $5 \mathrm{ng} / \mathrm{mL}$. Levels were at the lower limits (tacrolimus $5.2 \mathrm{ng} / \mathrm{mL}$ and everolimus $2.9 \mathrm{ng} / \mathrm{mL}$ ) under a daily dosage of $3 \mathrm{mg}$ tacrolimus and $2.5 \mathrm{mg}$ everolimus 1 week before the occurrence of diarrhea. During one episode of severe diarrhea, the patient was admitted to our hospital because of dehydration. The blood trough level of tacrolimus had increased from 5.2 to $21.6 \mathrm{ng} / \mathrm{mL}$, whereas everolimus had increased from 2.9 to $13.4 \mathrm{ng} / \mathrm{mL}$. By pausing both immunosuppressive medications, the levels decreased slowly, so that tacrolimus and everolimus could be continued in a reduced dosage after 2 days (tacrolimus daily dose $2 \mathrm{mg}$ and everolimus daily dose $1.5 \mathrm{mg}$ ). Ten days after the onset of diarrhea, the levels of both immunosuppressants returned to normal values under a reduced dosage. Furthermore, there was no need to increase the two immunosuppressive medications to the initial dose before diarrhea within 8 months after diarrhea had occurred.

\section{CASE 2}

The 13-year-old boy had to undergo heart transplantation 1998 because of dilated cardiomyopathy. Thirteen years later, he was on immunosuppressive therapy with mycophenolic acid (MPA) and everolimus. The target values of MPA were between 0.5 and $4 \mu \mathrm{g} / \mathrm{mL}$ and stayed within the target range before, during, and after diarrhea. It was possible to constantly achieve the target values of everolimus (between 5 and $8 \mathrm{ng} / \mathrm{mL}$ ) under a dosage of $4.75 \mathrm{mg}$ per day. During severe diarrhea, the everolimus level rose to $15.6 \mathrm{ng} / \mathrm{mL}$. Via dose reduction and normalization of the stool, simply under symptomatic therapy, the levels went back to normal in about 1 week. There was no need to change the dosage of MPA. Interestingly, as in the first case, it was not possible to return to the initial dose before diarrhea for more than 1 year.

In both cases, a correlation between the elevated values and medication or food was not detectable. There were no changes of medication, the chronological taking of everolimus related to food, or initiation of interacting drugs before the onset of diarrhea. In addition, there was no sign of reduced hepatic function that could have explained the elevated levels of everolimus. It was not possible to identify a specific cause for the diarrhea in the patients. However, a viral infection seems most likely.

There are reports on elevated tacrolimus levels during diarrhea (1-3). We detected the same effect with everolimus during diarrhea in two patients after heart transplantation.

The immunosuppressant everolimus has a low oral bioavailability of $16 \%$ (4). P-glycoprotein and cytochrome $\mathrm{P} 450$ are responsible for the absorption and metabolism of everolimus (4) as well as tacrolimus (2). Therefore, the reasons for elevated tacrolimus levels during diarrhea could accordingly apply to everolimus. Possible explanations are decreased activity of cytochrome P450 and P-glycoprotein (2) as well as a decreased hepatic blood flow with reduced hepatic metabolism (1). The apices of the enterocytes in the small intestine carry cytochrome P450. Lysis of the enterocytes by viral diarrhea leads to reduced activity of cytochrome P450 causing decreased intestinal metabolism of immunosuppressants with increased blood levels. In addition, a capacity overload could explain the concordant change of the immunosuppressant levels in case of a simultaneous taking of tacrolimus and everolimus.

According to the equivalent hypotheses concerning tacrolimus (2), the lower dosage for almost 1 year after diarrhea could be explained by the time required to normalize the activity of cytochrome P450 and P-glycoprotein.

For the evaluation of the concrete mechanism of elevated everolimus levels during diarrhea, further studies are necessary. However, regardless the mechanism responsible, it is important to know about the possible increase of everolimus during diarrhea. A frequent control of everolimus levels is recommended to avoid everolimusdependent adverse events.

Sarah M. Kohler Julia Birnbaum Alexandra Th. Fuchs Roxana Engmann Heinrich Netz Rainer Kozlik-Feldmann

Department of Pediatric Cardiology and Intensive Care Medicine Ludwig-Maximilians-University Munich Munich, Germany

The authors declare no funding or conflicts of interest. Address correspondence to: Sarah Kohler, M.D., Department of Pediatric Cardiology and Intensive Care Medicine, Ludwig-MaximiliansUniversity Munich, Marchioninistr. 15, 81377 Munich, Germany.

E-mail: Sarah.Kohler@med.uni-muenchen.de

S.M.K., J.B., and R.E. participated in the data analyses. S.M.K., A.T.F., H.N., and R.K.-F. participated in the writing of the article. Received 4 March 2013.

Accepted 6 August 2013

Copyright (C) 2013 by Lippincott Williams \& Wilkins ISSN: 0041-1337/13/9608-e62

DOI: $10.1097 /$ TP.0b013e3182a8611f

\section{REFERENCES}

1. Teisseyre J, Teisseyre M, Kalicinski P, et al. Elevated tacrolimus levels during diarrhea in children after liver transplantation. Transplant Proc 2003; 35: 2292.

2. Asano T, Nishimoto K, Hayakawa M. Increased tacrolimus trough levels in association with severe diarrhea, a case report. Transplant Proc 2004; 36: 2096.

3. Hochleitner BW, Bösmüller C, Nehoda H, et al. Increased tacrolimus levels during diarrhea. Transpl Int 2001; 14: 230.

4. Kirchner GI, Meier-Wiedenbach I, Manns MP. Clinical pharmacokinetics of everolimus. Clin Pharmacokinet 2004; 43: 83. 